\title{
BRAIN GENE EXPRESSION-DNA METHYLATION CORRELATION IN SUICIDE COMPLETERS: PRELIMINARY RESULTS
}

\author{
Brenda Cabrera-Mendoza ${ }^{1,2}$, José J. Martínez-Magaña ${ }^{1}$, Alma D. Genis-Mendoza ${ }^{1,3}$, \\ Nancy Monroy-Jaramillo ${ }^{4}$, Consuelo Walss-Bass ${ }^{5}$, Gabriel R. Fries $^{5}$, Fernando García-Dolores ${ }^{6}$, \\ Mauro López-Armenta ${ }^{6}$, Gonzalo Flores ${ }^{7}$, Rubén A. VÁzQuez-RoQue ${ }^{7}$, Humberto Nicolini ${ }^{1,8 *}$ \\ ${ }^{1}$ Genomics of Psychiatric and Neurodegenerative Diseases Laboratory, Instituto Nacional de Medicina Genómica, SSA, \\ Mexico City, Mexico; ${ }^{2}$ Combined Studies Program in Medicine MD/PhD, Faculty of Medicine, Universidad Nacional \\ Autónoma de México, Mexico City, Mexico; ${ }^{P}$ Psychiatric Care Services, Hospital Psiquiátrico Infantil "Juan N. Navarro," \\ SSA, Mexico City, Mexico; ${ }^{4}$ Department of Genetics, Instituto Nacional de Neurología y Neurocirugía "Manuel Velasco \\ Suárez," Mexico City, Mexico; ${ }^{5}$ Department of Psychiatry and Behavioral Sciences, University of Texas Health Science \\ Center at Houston, Texas, USA; ${ }^{6}$ Institute of Forensic Science, Mexico City, Mexico; ${ }^{7}$ Neuropsychiatry Laboratory, \\ Institute of Physiology, Benemérita Universidad de Puebla, Mexico; ${ }^{8}$ Grupo de Estudios Médicos y Familiares Carracci, \\ Mexico City, Mexico
}

\begin{abstract}
Background: Gene expression alterations have been implicated in suicide pathology. However, the study of the regulatory effect of DNA methylation on gene expression in the suicidal brain has been restricted to candidate genes. Objective: The objective of the study was to identify genes whose expression levels are correlated with DNA methylation in the prefrontal cortex of suicides. Methods: Postmortem prefrontal cortex samples from 21 suicides and six non-suicides were collected. Transcriptomic and DNA methylation profiles were evaluated with microarrays; cis correlations between gene expression and CpG methylation were screened. We then analyzed the presence of transcription factor (TF) binding sites (TFBS) at CpG sites correlated with gene expression. Gene expression of TFs involved in neurodevelopmental binding to predicted TFBS was determined in the BrainSpan database. Results: We identified $22 \mathrm{CpG}$ sites whose methylation levels correlated with gene expression in the prefrontal cortex of suicides. Genes annotated to identified CpG sites were involved in neurodevelopment (BBS4, NKX6-2, AXL, CTNND1, and MBP) and polyamine metabolism (polyamine oxidase [PAOX]). Such correlations were not detected in the nonsuicide group. Nine TFs (USF1, TBP, SF1, NRF1, RFX1, SP3, PKNOX1, MAZ, and POU3F2) showed differential expression in pre- and post-natal developmental periods, according to BrainSpan database. Conclusions: The integration of different omic technologies provided novel candidates for the investigation of genes whose expression is altered in the suicidal brain and their potential regulatory mechanisms. (REV INVEST CLIN. 2020;72(5):283-92)
\end{abstract}

Key words: Suicide. DNA methylation. Gene expression. Cerebral cortex. Microarrays. Transcription factors. Neurodevelopment.

*Corresponding author:

Humberto Nicolini

E-mail: hnicolini@inmegen.gob.mx
Received for publication: 9-09-2019

Approved for publication: 16-12-2019

DOI: $10.24875 /$ RIC.19003250

0034-8376 / (c) 2019 Revista de Investigación Clínica. Published by Permanyer. This is an open access article under the CC BY-NC-ND license (http://creativecommons.org/licenses/by-nc-nd/4.0/). 


\section{INTRODUCTION}

Suicide is one of the leading causes of death worldwide $^{1}$ and a growing health problem, as suicide rates have been increasing in many countries, including Mexico $^{2}$. Suicide is a complex behavior where several contributors interact, i.e., genetic, environmental, and sociocultural factors ${ }^{3,4}$. Among suicide-related factors, epigenetic changes seem to be particularly relevant in suicidal behavior, due to their mediating role in the complex interaction between genotype and environment ${ }^{5,6}$. Epigenetic modifications, such as DNA methylation, may regulate gene expression patterns in the brain, leading to the neurobiological abnormalities associated with suicidal behavior ${ }^{7}$.

Alterations in DNA methylation and gene expression have been widely evidenced in the suicidal brain and have been implicated in suicide pathology ${ }^{8-16}$. However, our understanding of the regulatory effect of DNA methylation in gene expression in suicides is scarce, since correlations between DNA hypermethylation and gene expression have been limited to candidate genes, such as tropomyosin-related kinase $B$ (TRKB), glucocorticoid receptor (GR), ornithine decarboxylase antizymes 1 and 2 (OAZ1 and OAZ2), quaking homolog $\mathrm{KH}$ domain RNA binding ( $Q K I)$, S-adenosylmethionine decarboxylase (AMD1), and arginase 2 $(A R G 2)^{17-21}$.

The integration of molecular traits at different levels is crucial to the elucidation of mechanisms leading to observed gene expression alterations in the suicidal brain. In this study, we aimed to identify CpG sites whose methylation levels are correlated with gene expression at a genome-wide level in the prefrontal cortex of suicides. The prefrontal cortex was selected for its analysis due to its importance in suicide, as there is extensive evidence of structural, cognitive, and molecular alterations in this brain area in individuals with suicidal behavior ${ }^{22-26}$.

The relevance of the bi-directional interactions between DNA methylation and transcription factors (TF) for regulating gene expression is noteworthy, as DNA methylation may inhibit TF binding and the interaction of TF with methylated CpG sites may translate DNA methylation signals into biological actions ${ }^{27,28}$. In this study, we performed a prediction of TF binding sites (TFBS) located in promoters related to the identified $\mathrm{CpG}$ sites. These are the preliminary results of an ongoing study on suicide genomics at the National Institute of Genomic Medicine in Mexico.

\section{METHODS}

\section{Samples}

Brain tissue samples from 21 male suicides and six male non-suicides were analyzed in this study. Male suicide samples have previously been described in Rodríguez-López et al. ${ }^{29}$, where the influence of genetic variants in gene expression and methylation profile in suicides was studied. Postmortem brain samples were collected at the Institute of Forensic Sciences in Mexico City, Mexico. Suicides died by selfinflicted injuries, and non-suicides presented a sudden non-suicidal self-inflicted death, as determined by the coroner.

Postmortem interval, which is the interval between the estimated time of death and sample collection, ranged from 8.31 to $23 \mathrm{~h}$ in the analyzed sample. Age and postmortem interval differences between suicides and non-suicides were evaluated by Wilcoxon rank-sum test. All individuals included in this study were Mexico City residents.

The present study was approved by the Ethical Committee for Human Research at the National Institute of Genomic Medicine and the Bioethics Committee of the Faculty of Medicine at the National Autonomous University of Mexico (UNAM). Tissue samples from the dorsolateral prefrontal cortex (Brodmann area 9) were sectioned during an autopsy protocol, following a previously described procedure ${ }^{30}$. Samples were stored in RNAlater at $-80^{\circ} \mathrm{C}$ until their use.

A consensus diagnosis of psychiatric and neurological disorders was made for each individual with information obtained from the coroner's records. Information contained in the coroner's records included (a) demographic information; (b) the autopsy report; (c) description of death circumstances; (d) results from the toxicology test, performed in both peripheral blood and brain tissue as part of the autopsy protocol; (e) police reports; ( $f$ ) medical and 
psychiatric notes, if available; (g) suicide notes; (h) testimonies from relatives and witnesses of suicide completers; and (i) the death certificate. Consensus diagnosis was performed according to the Diagnostic and Statistical Manual of Mental Disorders, Fifth Edition (DSM-5) criteria $^{31}$.

\section{Nucleic acids isolation}

Genomic DNA was isolated from $100 \mathrm{mg}$ of tissue with the QIAamp DNA Mini Kit from Qiagen ${ }^{\text {TM }}$. Meanwhile, RNA isolation was performed using the RNeasy kit from Qiagen ${ }^{\mathrm{Tm}}$. Isolation of both nucleic acids was performed according to the manufacturer's instructions. Then, DNA and RNA quantity and integrity were determined using the NanoDrop 2000 spectrometer (Thermo Fisher, Wilmington, DE, USA) and agarose gel electrophoresis, respectively. In addition, the RNA integrity number (RIN) of the samples was evaluated using Agilent Bioanalyzer. All samples were further processed as they complied with standard quality parameters, such as an adequate concentration (> $50 \mathrm{ng} / \mu \mathrm{l}$ ) and integrity. RNA samples had an $\mathrm{RIN}>6$.

\section{Microarray detection}

Isolated DNA was bisulfite-converted using the EZ DNA Methylation-Gold Kit (Zymo Research, Irvine, CA, USA) following the manufacturer's protocol. Then, bisulfite-converted DNA was hybridized into the Illumina Infinium Human Methylation 450K BeadChip (Illumina, San Diego, CA, USA) to evaluate the methylation profile of the samples.

For gene expression profiling, RNA samples were labeled into biotinylated cRNAs with the TargetAmp ${ }^{\text {TM }}$ Nano Labeling Kit (Epicentre). Then, samples were hybridized into the Illumina HumanHT-12 v4 BeadChip (Illumina, San Diego, CA, USA) microarray. The processing of both arrays was performed according to the established manufacturer's conditions and protocol and was scanned on an iScan Microarray Scanner (Illumina, San Diego, CA, USA) immediately after their respective protocol.

\section{DNA methylation data pre-processing}

IDAT files containing raw DNA methylation data were processed using minfi quality control and normalization options ${ }^{32}$. Microarray probes were filtered according to the following criteria: (i) detection $p>0.05$; (ii) with at least one SNP of minor allele frequency (MAF) $>5 \%$, according to the allele frequency estimated from 1000 Genomes Project samples ${ }^{33}$; and (iii) probes previously reported as cross-reactive ${ }^{33}$. A matrix of beta values was generated for correlation analysis.

\section{Gene expression data pre-processing}

Data pre-processing was conducted in the Gene Expression Module of GenomeStudio v2.0 software (IIlumina, USA). Microarray probes with a detection $p>$ 0.05 in more than $5 \%$ of the arrays were filtered out. Then, raw probe intensities were normalized and $\log (2)$-transformed with the quantile method ${ }^{34}$. A matrix of $\log (2)$ values was generated for correlation analysis. After quality control and pre-processing steps in each platform, we obtained a total of 8670 expressed genes and 468,922 CpG methylation sites, which were further analyzed for correlation between both molecular traits.

\section{Correlation analysis}

Correlation between DNA methylation beta values and gene expression values was tested with a linear regression adjusted by age and postmortem interval implemented in the R package MatrixEQTL ${ }^{35}$. To evaluate the effect of DNA methylation in both enhancers and promoters, distance thresholds for detection of CpG-gene pairs were set at $1 \mathrm{Mb}, 100 \mathrm{~kb}$, and $50 \mathrm{~kb}$ upstream or downstream from the transcription start site of the corresponding genes. A significant correlation was defined when its two-sided $p-<0.05$ after correction for multiple testing with the BenjaminiHochberg False Discovery Rate (FDR) method ${ }^{36}$. Then, the correlation of the CpG sites correlated with gene expression in the suicide group was tested in the nonsuicide control group.

To evaluate whether the gene expression-methylation correlations found in the suicide group were related to a comorbid psychiatric disorder, an additional correlation analysis was performed, separating suicides with major depression disorder $(n=8)$, which was the most common mental disorder in our sample, and suicides without mental comorbidities $(n=10)$. 
Table 1. Characteristics of subjects

\begin{tabular}{lcc}
\hline & Suicides & Non-suicides \\
\hline Number of individuals & 21 & 6 \\
Age (years) & $28.4 \pm 9.15$ & $29.33 \pm 8.09$ \\
Postmortem interval (hours) & $14.33 \pm 4.73$ & $15.53 \pm 3.78$ \\
Psychiatric disorder* & $8 / 3 / 10$ & $0 / 1 / 5$ \\
Substance reported in toxicology test** & $9 / 1 / 11$ & $0 / 0 / 6$ \\
Mechanism of death $^{\dagger}$ & $18 / 0 / 2 / 1$ & $2 / 4 / 0 / 0$ \\
\hline
\end{tabular}

Values indicate number of subjects unless otherwise indicated. Continuous data are presented as mean \pm standard deviation.

*Major depression disorder/Personality disorder/None. ${ }^{* *}$ Alcohol/Cocaine/None. ${ }^{\dagger}$ Asphyxia/Gunshot/Puncture wound/Trauma.

CpG sites were annotated with the IlluminaHumanMethylation450kmanifest package ${ }^{37}$. To gain insight into the key processes where genes correlated with CpG methylation are participating, gene ontology (GO) enrichment analysis was performed with the Database for Annotation, Visualization, and Integrated Discovery (DAVID), version $6.8^{38,39}$. GO terms with a modified Fisher exact $p<0.05$ (EASE score) were considered enriched ${ }^{40}$.

\section{Prediction of TFBS}

First, the DNA sequences from the $\mathrm{CpG}$ sites correlated with gene expression were obtained from the UCSC Genome Browser available at http://genome. ucsc.edu/ ${ }^{41}$. CpG site sequences were used as input in the Neural Network Promoter Prediction tool (NNPP) v2.2 (http://www.fruitfly.org/ index.html) to find possible transcription promoters and their sequence $^{42}$. Second, a TFBS prediction was performed in PROMO (http://alggen.Isi.upc.es/cgi-bin/promo) v8.3 for each transcription promoter sequence ${ }^{43,44}$. The search was restricted to eukaryotic factors and sites. Third, we evaluated the presence of gene expression changes in the TF with binding activity to the predicted TFBS in pre- and post-natal developmental periods with the query of developmental transcriptome reported in the BrainSpan database (www.brainspan.org $)^{45}$. To identify the specific stage where gene expression changes take place, additional comparisons between the following stages were performed: prenatal period (0-38 weeks), infancy (birth-18 months), childhood (19 months-11 years), adolescence (12-19 years), and adulthood ( $\geq 20$ years) in all brain regions.

\section{RESULTS}

Data from 21 male suicides and six male non-suicide controls were analyzed in this study. No significant differences were found between suicides and nonsuicides in terms of age $(p=0.5852)$ and postmortem interval $(p=0.5046)$. The sample characteristics are summarized in table 1 . A total of $22 \mathrm{CpG}$ sites showed a significant correlation with gene expression after correction for multiple testing in the suicide group. Among these, 17 pairs (77.27\%) were located within $1 \mathrm{Mb}$ upstream or downstream from the transcription start site. Meanwhile, three were found within $100 \mathrm{~kb}$ and two within $50 \mathrm{~kb}$ from the transcription start site.

Regarding location relative to genes, six CpG sites were located in promoter regions and three in enhancer regions. Regarding the location of $\mathrm{CpG}$ sites relative to CpG islands, 12 sites (54.54\%) were located inside CpG islands, six sites (27.27\%) on island shelves (four in north and two in south shelf), and four sites (18.19\%) were located on island shores (three in north and one in north shore). The list of CpG-gene pairs is shown in table 2 .

Functional analysis with DAVID indicated that five genes were significantly associated with central nervous system development (GO: 0007417, $\mathrm{p}=$ 0.0020 ). Of these genes, CTNND1 and AXL are involved in the VEGFA-VEGFR2 pathway (HSA-4420097, $p=0.0058$ ).

A total of 416 TF was predicted for the $\mathrm{CpG}$ sites identified in the present study. Of these, only 9e (USF1, TBP, SF1, NRF1, RFX1, SP3, PKNOX1, MAZ, 
Table 2. Significantly correlated CpG-gene pairs

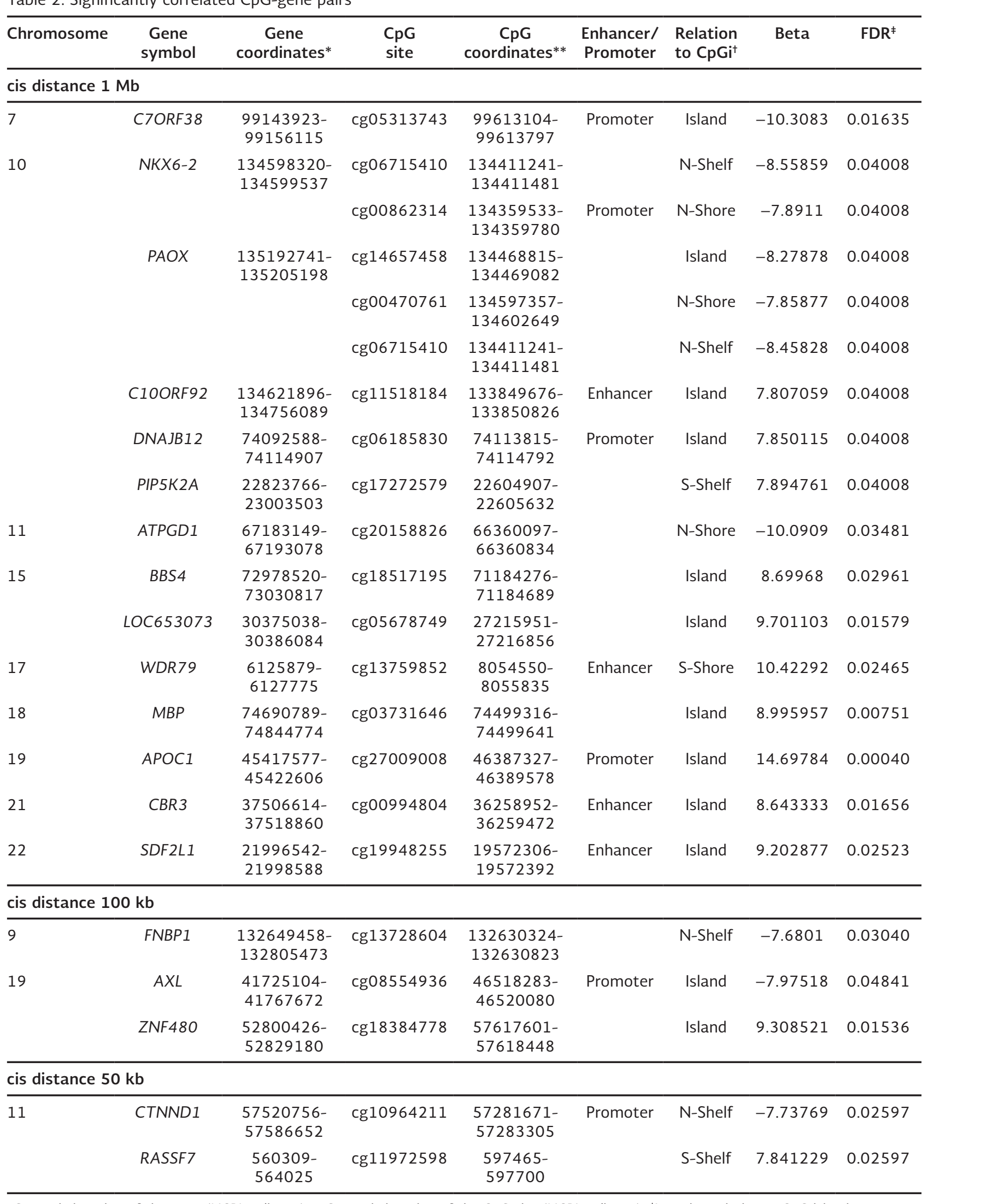

*Genomic location of the gene (NCBI37/hg19). **Genomic location of the CpG site (NCBI37/hg19). 'Location relative to CpG islands. FFDR-adjusted $\mathrm{p}$-value. 
Figure 1. Mean expression levels of transcription factors predicted to bind to promoter regions located in CpG sites correlated with gene expression in the prefrontal cortex of suicides during developmental stages.

Transcription factors expression across development stages

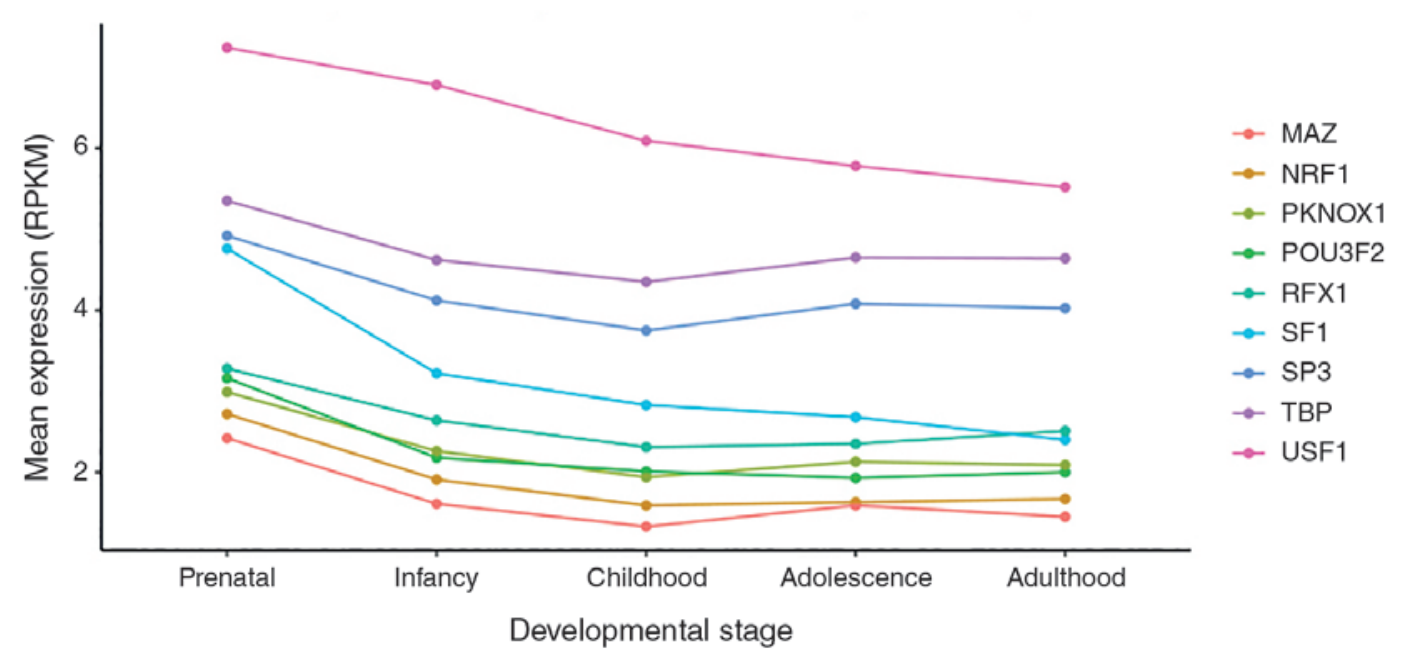

and POU3F2) showed differential expression, i.e., exhibited significantly different expression levels when comparing gene expression profile in pre- and postnatal developmental periods in the BrainSpan database. The gene expression pattern of these TF was analyzed across developmental stages, as shown in figure 1 . It was noteworthy that TF expression predicted to bind to promoter regions located in $\mathrm{CpG}$ sites was higher during prenatal stages and decreased as development progressed. Complete results of TF gene expression analysis in BrainSpan are shown in Supplementary table 1 .

The correlation between the $\mathrm{CpG}$ sites correlated with gene expression in the suicide group was tested in the non-suicide control group. However, this analysis did not show any significant correlation between the tested CpG site-gene pairs. The results of the correlation test in the non-suicide group are shown in Supplementary table 2 .

In addition, correlations were tested, separating suicides with major depression disorder $(n=8)$ from suicides without mental comorbidities $(n=10)$. Correlations were not significant in either group. The complete results of this sub-analysis are shown in Supplementary tables 3 and 4 .

\section{DISCUSSION}

To the best of our knowledge, this is the first wholegenome transcriptomic-methylomic correlation analysis in the dorsolateral prefrontal cortex of individuals who died by suicide. The previous studies have reported genes regulated by DNA methylation, mainly by candidate genes studies ${ }^{17-21}$. Correlations found in the suicide group were not detected in the nonsuicide group. Thus, our results provide new insights into the effects of DNA methylation on the brain gene expression of suicide.

Among the genes whose expression was found to be correlated to $\mathrm{CpG}$ sites, we identified genes involved in neurodevelopment, such as BBS4, NKX6-2, AXL, CTNND1, and MBP. This finding is consistent with the previous reports of misexpression of genes involved in central nervous system development in suicide $^{14,46}$. In addition, we identified a significant correlation with DNA methylation for the expression of genes involved in processes contributing to remodelings in the central nervous system, such as RASSF7 involved in cell proliferation and cytoskeletal organization 47,48; CTNND1 and MBP, which have been implicated in cell adhesion and migration ${ }^{49}$; and $M B P$, which encodes for a major constituent of the 
myelin sheath of oligodendrocytes ${ }^{50}$. Furthermore, the NKX6-2 gene has shown to have an important role in the generation of cortical interneurons and their differentiation ${ }^{51}$.

In addition, the prediction of TFBS in the identified CpG sites related to TF involved in neurodevelopment suggests a role of DNA methylation-TF interactions in the gene expression patterns observed in suicides. DNA-methylation-TF interactions have been related to the downregulation of gene expression ${ }^{52}$. However, recent studies have proven that such interactions are complex, and their effects are varied. Moreover, the effects seem to be bidirectional. For example, among the predicted TF found in our results, RFX1 modulates epigenetic modifications by recruiting the histone methyltransferase SUV39H1 $1^{53}$. Meanwhile, methylation in the core-promoter region of the chondromodulin-I gene regulates the binding of transcriptional activator $\mathrm{Sp}^{54}$. Thus, the effects of DNA methylation-TF interactions in neurodevelopment should be further studied.

Furthermore, the most remarkable TF expression changes were found between prenatal and postnatal developmental stages. The vulnerability of the brain to insults during the earliest developmental stages has been acknowledged as one of the factors associated with the susceptibility to neurodevelopmental and psychiatric disorders ${ }^{55}$. Therefore, our findings suggest a possible role for TF on neurodevelopmental alterations reported in the suicidal brain.

Our results suggest a role for DNA methylation and possibly TFs as well, in the regulation of neurodevelopmental gene expression, providing insight into the molecular mechanisms that might lead to neuroanatomical abnormalities in both gray and white matter reported in individuals with suicidal behavior ${ }^{56-58}$.

Our analysis suggests that polyamine oxidase (PAOX) expression may be functionally regulated by methylation. PAOX encodes for the PAOX, an enzyme that catalyzes the oxidation of $\mathrm{N} 1$-acetylspermidine and $\mathrm{N} 1$-acetylspermine products of spermidine/spermine $\mathrm{N} 1$-acetyltransferase (SAT1), producing spermidine and putrescine. Spermidine and putrescine levels found in the brain of suicide completers with a history of major depression were significantly higher compared to controls ${ }^{59}$. It has been hypothesized that this polyamine accumulation may be a consequence of decreased SAT1 expression ${ }^{60}$. However, the upregulation of PAOX provides a better explanation for the reported levels of polyamines in the suicidal brain, as both of its products appear to be increased in the suicidal brain.

Another possibility is that increased PAOX expression is a compensatory mechanism that decreases these levels ${ }^{60}$. PAOX appears to be controlled by substrate availability ${ }^{26}$; thus, its regulation at a transcriptional level by methylation might be an additional regulatory mechanism, as polyamine homeostasis is a highly regulated mechanism ${ }^{60}$. Alterations in the expression of polyamine-related genes have been consistently reported in the brain of suicide completers ${ }^{61}$. The regulation by DNA methylation in polyamine biosynthetic genes, such as $A M D 1, A R G 2, O A Z 1$, and $O A Z 2$, has been previously reported ${ }^{21}$. Therefore, the role of PAOX in suicide is worthy of exploring in future research.

Recently, an abnormal telomere length was identified in suicide completers ${ }^{62}$. The protein encoded by WDR79 is crucial in telomere synthesis, as it acts as a telomerase holoenzyme component, facilitating its assembly and translocation ${ }^{63}$. The modulation of the expression of WDR79 by DNA methylation as indicated by our results suggests a candidate mechanism, underpinning telomere alterations in suicide completers. Regarding other $\mathrm{CpG}$-gene pairs identified in this study, we found genes previously linked to mental disorders, such as PIP5K2A and ZNF480, that have been implicated in schizophrenia ${ }^{64,65}$. Future studies will determine whether these genes are implicated in suicide as well.

Some of the genes whose expressions were found to be correlated with DNA methylation in our study are involved in pathways previously reported as dysregulated in suicide, as in the case of neurodevelopment and polyamines. The findings of this study improve our understanding of the regulatory mechanisms that lead to previously reported abnormalities in the suicidal brain. Furthermore, our study provided novel candidates for the investigation of genes regulated by DNA methylation in completed suicides.

It is noteworthy that this study focused on the Mexican population. Most postmortem suicide studies 
have focused on the Caucasian population, and studies in the Latino population regarding this subject are very scarce. Since environmental factors influencing epigenetic modifications vary among different populations, and specifically Mexican Mestizo populations have shown high genomic variability ${ }^{66}$, the study of individuals with diverse ethnic backgrounds will allow the identification of population-specific alterations in the suicidal brain. The inclusion of socio-demographic and clinical variables will allow the identification of factors associated with the observed molecular traits. In addition to DNA methylation, it is important to consider the influence of other factors in gene expression, for example, other epigenetic factors such as histone modification may influence this phenotype and should be further studied.

Limitations of the present study must be acknowledged. First, the relatively small sample size and that the analysis was restricted to male individuals. Therefore, future research with larger samples and individuals of both sexes is necessary to confirm our results and their association with suicide. Second, our sample included individuals with different psychiatric disorders, which may represent a confounding factor in our analysis. Correlations were not significant when separating suicides with major depression disorder from suicides without comorbid mental disorders, as well as in non-suicides. This lack of correlation might be due to the small sample size found in each group. The tendency of a cg06715410/ NKX6-2 pair to correlate with an FDR corrected $p$ value of 0.07793 in the non-suicide group is noteworthy. This finding encourages correlation testing in larger samples of suicides and non-suicides with and without mental disorders for identifying correlations specific to each condition. Future studies should include groups of suicides and non-suicides with mental disorders in an adequate number for estimating the effect of the comorbid mental disorder in gene expression and methylation. As part of an ongoing project, which started in 2018, we expect to collect more samples and to overcome the aforementioned limitations.

\section{ACKNOWLEDGMENTS}

We would like to thank the staff of the Microarray Unit at INMEGEN for their technical support. Brenda
Cabrera-Mendoza is a doctoral student from the Plan of Combined Studies in Medicine (PECEM) at the National Autonomous University of Mexico and was supported by CONACYT (No. 622945). José Jaime Martínez-Magaña is a doctoral student from the Biomedical Sciences Program at the Juárez Autonomous University of Tabasco and was supported by CONACyT. The authors also thank Professor Robert Simpson for editing the English language style.

\section{SUPPLEMENTARY DATA}

Supplementary data are available at Revista de Investigación Clínica online (www.clinicalandtranslationalinvestigation.com). These data are provided by the corresponding author and published online for the benefit of the reader. The contents of supplementary data are the sole responsibility of the authors.

\section{REFERENCES}

1. World Health Organization. Preventing Suicide: A Global Imperative. Geneva: World Health Organization; 2015. Available from: http://www.who.int/mental_health/suicide-prevention/ world_report_2014/en. [Last cited on 2019 Aug 14].

2. Instituto Nacional de Estadística y Geografía. Estadísticas a Propósito del día Mundial Para la Prevención del Suicidio. México: Instituto Nacional de Estadística y Geografía; 2018. Available from: https://www.inegi.org.mx/contenidos/saladeprensa/aproposito/2018/suicidios2018_Nal.pdf. [Last cited on 2019 Aug 14].

3. Mann JJ. Neurobiology of suicidal behaviour. Nat Rev Neurosci. 2003;4:819-28.

4. Turecki G, Ernst C, Jollant F, Labonté B, Mechawar N. The neurodevelopmental origins of suicidal behavior. Trends Neurosci. 2012;35:14-23.

5. Schneider E, El Hajj N, Müller F, Navarro B, Haaf T. Epigenetic dysregulation in the prefrontal cortex of suicide completers. Cytogenet Genome Res. 2015;146:19-27.

6. Murphy TM, Crawford B, Dempster EL, Hannon E, Burrage J, Turecki G, et al. Methylomic profiling of cortex samples from completed suicide cases implicates a role for PSORS1C 3 in major depression and suicide. Transl Psychiatry. 2017;7:e989.

7. Labonté B, Suderman M, Maussion G, Lopez JP, Navarro-Sánchez $\mathrm{L}$, Yerko V, et al. Genome-wide methylation changes in the brains of suicide completers. Am J Psychiatry. 2013;170:511-20.

8. Fiori LM, Turecki G. Approaches and findings from gene expression profiling studies of suicide. In: Dwivedi $Y$, editor. The Neurobiological Basis of Suicide. Ch. 12. Boca Raton, FL: CRC Press/ Taylor and Francis; 2012.

9. Pandey GN, Dwivedi Y. What can post-mortem studies tell us about the pathoetiology of suicide? Future Neurol. 2010; 5:701-20.

10. Kim S, Choi KH, Baykiz AF, Gershenfeld HK. Suicide candidate genes associated with bipolar disorder and schizophrenia: an exploratory gene expression profiling analysis of post-mortem prefrontal cortex. BMC Genomics. 2007;8:413.

11. Lalovic A, Klempan T, Sequeira A, Luheshi G, Turecki G. Altered expression of lipid metabolism and immune response genes in the frontal cortex of suicide completers. J Affect Disord. 2010; 120:24-31. 
12. Sequeira A, Klempan T, Canetti L, ffrench-Mullen J, Benkelfat C, Rouleau GA, et al. Patterns of gene expression in the limbic system of suicides with and without major depression. Mol Psychiatry. 2007:12:640-55.

13. Sequeira A, Mamdani F, Ernst C, Vawter MP, Bunney WE, Lebel $V$, et al. Global brain gene expression analysis links glutamatergic and GABAergic alterations to suicide and major depression. PLoS One. 2009;4:e6585.

14. Thalmeier A, Dickmann M, Giegling I, Schneider B, M Hartmann A, Maurer K, et al. Gene expression profiling of post-mortem orbitofrontal cortex in violent suicide victims. Int J Neuropsychopharmacol. 2008;11:217-28.

15. Bani-Fatemi A, Howe AS, De Luca V. Epigenetic studies of suicidal behavior. Neurocase. 2015;21:134-43.

16. Haghighi F, Xin Y, Chanrion B, O'Donnell AH, Ge Y, Dwork AJ, et al. Increased DNA methylation in the suicide brain. Dialogues Clin Neurosci. 2014;16:430-8

17. Ernst C, Deleva V, Deng X, Sequeira A, Pomarenski A, Klempan $T$, et al. Alternative splicing, methylation state, and expression profile of tropomyosin-related kinase B in the frontal cortex of suicide completers. Arch Gen Psychiatry. 2009;66:22-32.

18. Klempan TA, Ernst C, Deleva V, Labonte B, Turecki G. Characterization of QKI gene expression, genetics, and epigenetics in suicide victims with major depressive disorder. Biol Psychiatry. 2009:66:824-31.

19. Labonte B, Yerko V, Gross J, Mechawar N, Meaney MJ, Szyf $M$, et al. Differential glucocorticoid receptor exon $1(B), 1(C)$, and $1(\mathrm{H})$ expression and methylation in suicide completers with a history of childhood abuse. Biol Psychiatry. 2012; 72:41-8.

20. McGowan PO, Sasaki A, D’Alessio AC, Dymov S, Labonté B, Szyf $M$, et al. Epigenetic regulation of the glucocorticoid receptor in human brain associates with childhood abuse. Nat Neurosci. 2009;12:342-8.

21. Gross JA, Fiori LM, Labonté B, Lopez JP, Turecki G. Effects of promoter methylation on increased expression of polyamine biosynthetic genes in suicide. J Psychiatr Res. 2013;47:513-9.

22. Raust A, Slama F, Mathieu F, Roy I, Chenu A, Koncke D, et al. Prefrontal cortex dysfunction in patients with suicidal behavior. Psychol Med. 2007;37:411-9.

23. Richard-Devantoy S, Berlim MT, Jollant F. A meta-analysis of neuropsychological markers of vulnerability to suicidal behavior in mood disorders. Psychol Med. 2014;44:1663-73.

24. Ding $Y$, Lawrence N, Olié E, Cyprien F, le Bars E, Bonafé $A$, et al. Prefrontal cortex markers of suicidal vulnerability in mood disorders: a model-based structural neuroimaging study with a translational perspective. Transl Psychiatry. 2015:5:e516.

25. van Heeringen C, Bijttebier S, Godfrin K. Suicidal brains: a review of functional and structural brain studies in association with suicidal behaviour. Neurosci Biobehav Rev. 2011:35:688-98.

26. Turecki G. The molecular bases of the suicidal brain. Nat Rev Neurosci. 2014;15:802-16.

27. Yin Y, Morgunova E, Jolma A, Kaasinen E, Sahu B, Khund-Sayeed $\mathrm{S}$, et al. Impact of cytosine methylation on DNA binding specificities of human transcription factors. Science. 2017;356: eaaj2239.

28. Zhu H, Wang G, Qian J. Transcription factors as readers and effectors of DNA methylation. Nat Rev Genet. 2016;17: 551-65.

29. Rodríguez-López ML, Martínez-Magaña JJ, Cabrera-Mendoza B, Genis-Mendoza AD, García-Dolores F, López-Armenta M, et al. Exploratory analysis of genetic variants influencing molecular traits in cerebral cortex of suicide completers. Am J Med Genet B Neuropsychiatr Genet. 2020;183:26-37.

30. Cabrera B, Monroy-Jaramillo N, Fries GR, Mendoza-Morales RC, García-Dolores F, Mendoza-Larios A, et al. Brain gene expression pattern of subjects with completed suicide and comorbid substance use disorder. Mol Neuropsychiatry. 2019;5:60-73.

31. American Psychiatric Association. Diagnostic and Statistical Manual of Mental Disorders. 5th ed. Arlington: American Psychiatric Association; 2013.

32. Aryee MJ, Jaffe AE, Corrada-Bravo H, Ladd-Acosta C, Feinberg AP, Hansen KD, et al. Minfi: a flexible and comprehensive Bioconductor package for the analysis of infinium DNA methylation microarrays. Bioinformatics. 2014;30:1363-9.

33. Chen YA, Lemire M, Choufani S, Butcher DT, Grafodatskaya D, Zanke BW, et al. Discovery of cross-reactive probes and polymorphic CpGs in the illumina infinium human methylation 450 microarray. Epigenetics. 2013;8:203-9.
34. Smyth GK, Speed T. Normalization of cDNA microarray data. Methods. 2003;31:265-73.

35. Shabalin AA. Matrix eQTL: ultra fast eQTL analysis via large matrix operations. Bioinformatics. 2012;28:1353-8

36. Benjamini $Y$, Hochberg $Y$. Controlling the false discovery rate a practical and powerful approach to multiple testing. J Roy Stat Soc B Met. 1995; 57:289-300.

37. Hansen KD, Aryee M. Illumina Human Methylation 450k Manifest: annotation for Illumina's 450k Methylation Arrays. R Package Version 0.4.0. Software; 2012.

38. Huang da W, Sherman BT, Lempicki RA. Systematic and integrative analysis of large gene lists using DAVID bioinformatics resources. Nat Protoc 2009:4:44-57.

39. Huang da W, Sherman BT, Lempicki RA. Bioinformatics enrichment tools: paths toward the comprehensive functional analysis of large gene lists. Nucleic Acids Res. 2009;37:1-13.

40. Hosack DA, Dennis G Jr., Sherman BT, Lane HC, Lempicki RA. Identifying biological themes within lists of genes with EASE. Genome Biol. 2003:4:R70

41. Kent WJ, Sugnet CW, Furey TS, Roskin KM, Pringle TH, Zahler AM, et al. The human genome browser at UCSC. Genome Res. 2002:12:996-1006.

42. Reese M, Harris NL, Eeckman FH. Large scale sequencing specific neural networks for promoter and splice site recognition. In: Hunter L, Klein TE, editors. Biocomputing Proceedings of the 1996 Pacific Symposium; 1996 Jan 2-7. Singapore: World Scientific Co.; 1996. p. 737-8.

43. Messeguer X, Escudero R, Farré D, Núñez O, Martínez J, Albà MM. PROMO: detection of known transcription regulatory elements using species-tailored searches. Bioinformatics. 2002; 18:333-4

44. Farré $D$, Roset $R$, Huerta $M$, Adsuara JE, Roselló L, Albà MM, et al. Identification of patterns in biological sequences at the ALGGEN server: PROMO and MALGEN. Nucleic Acids Res. 2003 31:3651-3

45. Tebbenkamp AT, Willsey AJ, State MW, Sestan N. The developmental transcriptome of the human brain: implications for neurodevelopmental disorders. Curr Opin Neurol. 2014;27: 149-56.

46. Zhurov V, Stead JD, Merali Z, Palkovits M, Faludi G, Schild-Poulter $C$, et al. Molecular pathway reconstruction and analysis of disturbed gene expression in depressed individuals who died by suicide. PLoS One. 2012;7:e47581.

47. Kumaraswamy A, Mamidi A, Desai P, Sivagnanam A, Perumalsamy LR, Ramakrishnan C, et al. The non-enzymatic RAS effector RASSF7 inhibits oncogenic c-Myc function. J Biol Chem. 2018:293:15691-705

48. Recino A, Sherwood V, Flaxman A, Cooper WN, Latif F, Ward A, et al. Human RASSF7 regulates the microtubule cytoskeleton and is required for spindle formation, Aurora B activation and chromosomal congression during mitosis. Biochem J. 2010 430:207-13

49. Ishiyama N, Lee SH, Liu S, Li GY, Smith MJ, Reichardt LF, et al. Dynamic and static interactions between p120 catenin and Ecadherin regulate the stability of cell-cell adhesion. Cell. 2010; $141: 117-28$

50. Boggs JM. Myelin basic protein: a multifunctional protein. Cell Mol Life Sci. 2006;63:1945-61

51. Sousa VH, Miyoshi G, Hjerling-Leffler J, Karayannis T, Fishell G. Characterization of Nkx6-2-derived neocortical interneuron lineages. Cereb Cortex. 2009;19 Suppl 1:i1-10.

52. Wang G, Luo X, Wang J, Wan J, Xia S, Zhu H, et al. MeDReaders: a database for transcription factors that bind to methylated DNA. Nucleic Acids Res. 2018;46:D146-51

53. Zhao M, Wu X, Zhang Q, Luo S, Liang G, Su Y, et al. RFX1 regulates CD70 and CD11a expression in lupus T cells by recruiting the histone methyltransferase SUV39H1. Arthritis Res Ther. 2010;12:R227.

54. Aoyama T, Okamoto T, Nagayama S, Nishijo K, Ishibe T, Yasura $\mathrm{K}$, et al. Methylation in the core-promoter region of the chondromodulin-I gene determines the cell-specific expression by regulating the binding of transcriptional activator Sp3. J Biol Chem. 2004;279:28789-97.

55. van den Bergh BRH, Dahnke R, Mennes M. Prenatal stress and the developing brain: risks for neurodevelopmental disorders. Dev Psychopathol. 2018;30:743-62.

56. Balcioglu YH, Kose S. Neural substrates of suicide and suicidal behaviour: from a neuroimaging perspective. Psychiatr Clin Psychopharmacol. 2018;28:314-28. 
57. Schnieder TP, Trencevska I, Rosoklija G Stankov A, Mann J], Smiley J, et al. Microglia of prefrontal white matter in suicide. J Neuropathol Exp Neurol. 2014;73:880-90.

58. Cox Lippard ET, Johnston JA, Blumberg HP. Neurobiological risk factors for suicide: insights from brain imaging. Am J Prev Med. 2014;47:S152-62.

59. Chen GG, Fiori LM, Moquin L, Gratton A, Mamer O, Mechawar $\mathrm{N}$, et al. Evidence of altered polyamine concentrations in cerebral cortex of suicide completers. Neuropsychopharmacology. 2010;35:1477-84.

60. Fiori LM, Turecki G. Implication of the polyamine system in mental disorders. J Psychiatry Neurosci. 2008;33:102-10.

61. Gross JA, Turecki G. Suicide and the polyamine system. CNS Neurol Disord Drug Targets. 2013;12:980-8.

62. Otsuka I, Izumi T, Boku S, Kimura A, Zhang Y, Mouri K, et al. Aberrant telomere length and mitochondrial DNA copy number in suicide completers. Sci Rep. 2017;7:3176.
63. Venteicher AS, Abreu EB, Meng Z, McCann KE, Terns RM, Veenstra TD, et al. A human telomerase holoenzyme protein required for Cajal body localization and telomere synthesis. Science. 2009:323:644-8.

64. He Z, Li Z, Shi Y, Tang W, Huang K, Ma G, et al. The PIP5K2A gene and schizophrenia in the Chinese population a case-control study. Schizophr Res. 2007;94:359-65.

65. Jouan L, Girard SL, Dobrzeniecka S, Ambalavanan A, Krebs MO Joober R, et al. Investigation of rare variants in LRP1, KPNA1, ALS2CL and ZNF480 genes in schizophrenia patients reflects genetic heterogeneity of the disease. Behav Brain Funct. 2013;9:9.

66. Silva-Zolezzi I, Hidalgo-Miranda A, Estrada-Gil J, Fernandez-Lopez JC, Uribe-Figueroa L, Contreras A, et al. Analysis of genomic diversity in Mexican Mestizo populations to develop genomic medicine in Mexico. Proc Natl Acad Sci U S A. 2009; 106:8611-6. 